\title{
SOME RESULTS ON THE ASYMPTOTIC BEHAVIOR OF LINEAR SYSTEMS
}

\author{
M. MARCUS
}

1. Introduction. We consider first in $\$ 2$ the asymptotic behavior as $t \rightarrow \infty$ of the solutions of the vector-matrix differential equation

$$
\dot{x}=\{A+B(t)\} x,
$$

where $A$ is a constant $n$-square complex matrix, $B(t)$ a continuous complex valued $n$-square matrix defined on $[0, \infty)$, and $x$ a complex $n$-vector.

It is readily shown (4) that the asymptotic behavior of solutions to (1.1) can be made to depend on the functions $\lambda_{M}\left\{A+A^{*}+B(t)+B^{*}(t)\right\}$ and $\lambda_{m}\left\{A+A^{*}+B(t)+B^{*}(t)\right\}$ where $A^{*}=\bar{A}^{\prime}$ and $\lambda_{M}, \lambda_{m}$ are respectively the maximum and minimum eigenvalues of the indicated Hermitian matrix. We recapitulate this brief calculation in $\S 2$.

There are two types of theorems concerning (1.1) in the sequel: (i) $A$ arbitrary with hypotheses on the eigenvalues of $\left(A+A^{*}\right)$; (ii) $A$ triangular with hypotheses on the real parts of the eigenvalues of $A$. In both (i) and (ii) less than the absolute integrability of the functions $B_{i j}(t)$ is required (1, pp. 32-63).

In $\S 3$ we discuss the behavior as $t \rightarrow \infty$ of solutions to the equation

$$
\dot{x}=\{A(t)+B(t)\} x,
$$

in which the entries of $A(t)$ are continuous complex-valued almost-periodic functions. The main result concerning (1.2) depends on a theorem of Favard which will be stated. In this case, however, it becomes necessary to assume the absolute integrability of all entries of $B(t)$.

We set

$$
\|x\|^{2}=\sum_{i=1}^{n}\left|x_{i}\right|^{2}
$$

boundedness refers to this norm. Also let $\Re(X)=(X+\bar{X}) / 2$ and $\Im(X)=$ $(X-\bar{X}) / 2 i .\|X\|^{2}=$ trace $\left(X X^{*}\right), \alpha^{\prime}=$ transpose of $\alpha$. We note the following two elementary results that are subsequently used:

\section{If $X$ and $Y$ are Hermitian $n$-square matrices then}

$$
\begin{aligned}
& \lambda_{M}(X+Y) \leqslant \lambda_{M}(X)+\lambda_{M}(Y), \\
& \lambda_{m}(X+Y) \geqslant \lambda_{m}(X)+\lambda_{m}(Y) .
\end{aligned}
$$

Received October 4, 1954; in revised form April 20, 1955. 
This follows immediately upon noting that $X+Y$ is Hermitian and

$$
\begin{aligned}
\lambda_{M}(X+Y) & =\max _{t=1} z^{*}(X+Y) z \leqslant \max _{t=1} z^{*} X z+\max _{t=1} z^{*} Y z \\
& =\lambda_{M}(X)+\lambda_{M}(Y),
\end{aligned}
$$

where $t=\|z\|^{2}$. Similarly for (1.4).

The following well-known device is due to O. Perron.

II. If $X$ has eigenvalues $\lambda_{1}, \ldots, \lambda_{n}$ then for any $\epsilon>0$ there exists a matrix $D(\epsilon)$ similar to $X$ such that $D_{i i}(\epsilon)=\lambda_{i}$ and $\left|D_{i j}(\epsilon)\right|<\epsilon$ for $i \neq j$.

For assume $X$ is in Jordan form, set

$$
H=\left(\begin{array}{cccc}
1 & 0 & \ldots & 0 \\
0 & \epsilon . & & 0 \\
\cdot & & \cdot & \cdot \\
\cdot & & \cdot & \cdot \\
\cdot & & \cdot & 0 \\
0 & \ldots & 0 & \epsilon^{n-1}
\end{array}\right)
$$

and note that

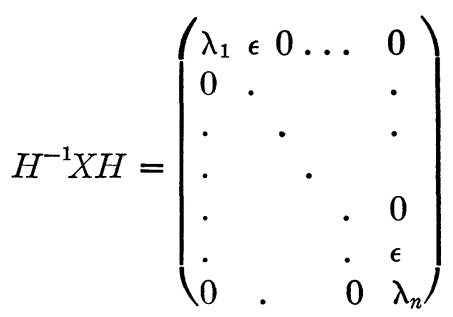

2. The equation (1.1). In discussing (1.1) we, of course, omit the trivial solution $x(t)=0$. We assume that the starting time of every solution is $t_{0}=0$ since any solution $x(t)$ with starting time $t_{0}>0$ may be continued over $\left[0, t_{0}\right]$.

Theorem 1. Consider (1.1) with A arbitrary. Assume

$$
\lambda_{M}\left(A+A^{*}\right)=\omega
$$

and there exists $L$ such that $t \geqslant L$ implies either (a):

$$
\frac{1}{t} \int_{0}^{t} \max _{i} \Re\left\{B_{i i}(s)\right\} d s \leqslant-\frac{1}{2} \omega
$$

$$
\int_{0}^{\infty}\left|\Re\left\{B_{i j}(s)\right\}\right| d s<\infty, \int_{0}^{\infty}\left|\Im\left\{B(s)-B^{\prime}(s)\right\}_{i j}\right| d s<\infty
$$

for $i \neq j$, or $(\mathrm{b})$ :

(2.4) $\frac{1}{t} \int_{0}^{t}\left(\max _{i} \Re\left\{B_{i i}(s)\right\}+\sum_{i \neq j}\left|\Re\left\{B_{i j}(s)\right\}\right|+\left|\Im\left\{B(s)-B^{\prime}(s)\right\}_{i j}\right|\right) d s$ 
then in both cases every solution of (1.1) is uniformly bounded as $t \rightarrow \infty$. If in either (2.2) or (2.4) the left sides are bounded strictly below $-\frac{1}{2} \omega$, then every solution converges to 0 as $t \rightarrow \infty$.

Proof. Taking the inner product on the left with $x^{*}$ in (1.1) we obtain

and

$$
x^{*} \dot{x}=x^{*}\{A+B(t)\} x
$$

$$
\frac{d}{d t}\|x\|^{2}=x^{*} \dot{x}+\dot{x}^{*} x=x^{*}\left\{A+A^{*}+B(t)+B^{*}(t)\right\} x .
$$

The matrix on the right in (2.6) is Hermitian for all $t$ and hence let $U(t)$ be a unitary matrix reducing it to canonical form. The substitution $x=U(t) z$ then yields

$$
\begin{aligned}
\frac{d}{d t}\|z\|^{2} & =z^{*} \text { diagonal } \lambda_{i}\left\{A+A^{*}+B(t)+B^{*}(t)\right\} z \\
& =\sum_{i=1}^{n} \lambda_{i}\left|z_{i}\right|^{2}=\|z\|^{2} \sum_{i=1}^{n} \lambda_{i} \delta_{i}
\end{aligned}
$$

where $\delta_{i}=\left|z_{i}\right|^{2} /|| z||^{2}, \sum \delta_{i}=1,0 \leqslant \delta_{i} \leqslant 1$. Integrating we obtain

$$
\|z(t)\|^{2}=\left\|z_{0}\right\|^{2} \exp \left(\int_{0}^{t} \sum_{i=1}^{n} \lambda_{i} \delta_{i} d s\right) .
$$

We use (1.2) to obtain

$$
\begin{gathered}
\sum_{i=1}^{n} \lambda_{i}\left\{A+A^{*}+B(t)+B^{*}(t)\right\} \delta_{i} \leqslant \lambda_{M}\left\{A+A^{*}+B(t)+B^{*}(t)\right\} \\
\leqslant \lambda_{M}\left(A+A^{*}\right)+\lambda_{M}\left\{B(t)+B^{*}(t)\right\} .
\end{gathered}
$$

Now let $m(s)$ be the unit eigenvector of $\left\{B(s)+B^{*}(s)\right\}$ such that

$$
\lambda_{M}\left\{B(s)+B^{*}(s)\right\}=m^{*}(s)\left\{B(s)+B^{*}(s)\right\} m(s)
$$

for $0 \leqslant s \leqslant \infty$. Also, setting $B(s)=U(s)+i V(s)$ and $m(s)=\alpha(s)+i \phi(s)$, (2.9) becomes

$$
\begin{gathered}
\text { (2.10) } \lambda_{M}\left(B(s)+B^{*}(s)\right)=\Re\left(m^{*}(s)\left\{B(s)+B^{*}(s)\right\} m(s)\right) \\
=\Re\left[\left\{\alpha^{\prime}(s)-i \phi^{\prime}(s)\right\}\left(U(s)+U^{\prime}(s)+i\left\{V(s)-V^{\prime}(s)\right\}\right)(\alpha(s)+i \phi(s))\right] \\
=2\left(\alpha^{\prime}(s) U(s) \alpha(s)+\phi^{\prime}(s) U(s) \phi(s)+\alpha^{\prime}(s)\left\{V^{\prime}(s)-V(s)\right\} \phi(s)\right) \\
=2 \sum_{i=1}^{n} U_{i i}(s)\left\{\alpha_{i}{ }^{2}(s)+\phi_{i}{ }^{2}(s)\right\}+2 \sum_{i \neq j} U_{i j}(s)\left\{\alpha_{i}(s) \alpha_{j}(s)+\phi_{i}(s) \phi_{j}(s)\right\} \\
+2 \sum_{i \neq j}\left\{V_{j i}(s)-V_{i j}(s)\right\} \alpha_{i}(s) \phi_{j}(s) .
\end{gathered}
$$

Now

$$
m^{*}(s) m(s)=\sum_{i=1}^{n}\left\{\alpha_{i}{ }^{2}(s)+\phi_{i}{ }^{2}(s)\right\}=1
$$

and we obtain 
(2.11)

$$
\begin{aligned}
\lambda_{M}\left\{B(s)+B^{*}(s)\right\} \leqslant 2 \max U_{i i}(s)+4 \sum_{i \neq j}\left|U_{i j}(s)\right| & +2 \sum_{i \neq j}\left|V_{i j}(s)-V_{j i}(s)\right| .
\end{aligned}
$$

We conclude from (2.7), (2.8) and (2.11) that

$$
\begin{aligned}
\|z(t)\|^{2} & \leqslant\left\|z_{0}\right\|^{2} \exp \left(\omega t+2 \int_{0}^{t} \max U_{i i}(s) d s\right. \\
& \left.+4 \int_{0}^{t} \sum_{i \neq j}\left|U_{i j}(s)\right| d s+2 \int_{0}^{t} \sum_{i \neq j}\left|V_{i j}(s)-V_{j i}(s)\right| d s\right) .
\end{aligned}
$$

In case (a), by (2.3), we select $K>0$ such that

$$
\|z(t)\|^{2} \leqslant K|| z_{0} \|^{2} \exp \left(t\left\{\omega+\frac{2}{t} \int_{0}^{t} \max U_{i i}(s) d s\right\}\right),
$$

and the result follows from (2.2). Case (b) is analogous with the use of (2.4) and (2.12).

Theorem 2. Consider

$$
\dot{x}=\{T+B(t)\} x
$$

and assume

(2.15) $T$ is triangular, $T_{i j}=0$ for $j<i$, and $\max \Re\left\{\lambda_{i}(T)\right\}=\omega$,

$$
\int_{0}^{\infty}\left|B_{i j}(t)\right| d t<\infty, \quad i \neq j,
$$

then every solution of (2.14) converges to 0 as $t \rightarrow \infty$.

Proof. Let $x=H y$ where

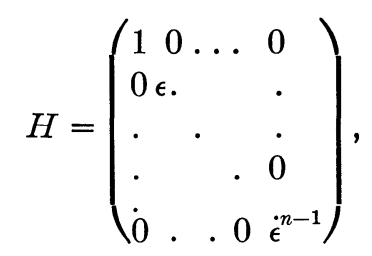

$\epsilon \neq 0$ then (2.14) becomes $\dot{y}=\{D+C(t)\} y$ with $D=H^{-1} T H$, $C(t)=H^{-1} B(t) H$. Proceeding as above we obtain

$$
\|z(t)\|^{2}=\left\|z_{0}\right\|^{2} \exp \left(\int_{0}^{t} \sum_{i=1}^{n} \lambda_{i}\left\{D+D^{*}+C(s)+C^{*}(s)\right\} \delta_{i} d s\right)
$$

where $z$ is the unitary transform of $y$. Now

$$
\left(D+D^{*}\right)_{i j}= \begin{cases}2 \Re\left\{\lambda_{i}(T)\right\}, & i=j, \\ \epsilon^{j-i} T_{i j,}, & i<j, \\ \epsilon^{i-j} \bar{T}_{j i}, & j<i,\end{cases}
$$


and $C_{i j}(s)=\epsilon^{i-j} B_{i j}(s)$. By (2.16) and (2.18) there exists such a constant $K$ that

$$
\|x(t)\|^{2} \leqslant K\left\|x_{0}\right\|^{2} \exp \left(t \lambda_{M}\left(D+D^{*}\right)+2 \int_{0}^{t} \max \Re\left\{B_{i i}(s)\right\} d s\right) ;
$$

but since the eigenvalues of a matrix are continuous functions of the entries, $\lambda_{M}\left(D+D^{*}\right)$ can be made to differ arbitrarily little from $2 \max \Re\left(\lambda_{i}(T)\right)=2 \omega$ by choosing $\epsilon$ sufficiently small. (2.17) completes the argument.

The divergence theorems follow analogously. We omit proofs.

Theorem 3. Consider (1.1). Assume

$$
\lambda_{m}\left(A+A^{*}\right)=\omega
$$

and

$$
\begin{aligned}
\liminf _{t \rightarrow \infty} \frac{1}{t} \int_{0}^{t}\left(\min \Re\left\{B_{i i}(s)\right\}-2 \sum_{i \neq j}\left|\Re\left\{B_{i j}(s)\right\}\right|\right. \\
\left.\quad-\sum_{i \neq j}\left|\Im\left\{B(s)-B^{\prime}(s)\right\}_{i j}\right|\right) d s>-\frac{1}{2} \omega ;
\end{aligned}
$$

then every solution of (2.1) diverges to $\infty$ as $t \rightarrow \infty$.

Theorem 4. Consider (2.14). Assume (2.16), $T$ triangular, $\min \Re\left(\lambda_{i}(T)\right)=\omega$ and

$$
\liminf _{t \rightarrow \infty} \frac{1}{t} \int_{0}^{t} \min \Re\left(B_{i i}(s)\right) d s>-\omega ;
$$

then every solution of (2.14) diverges to $\infty$ as $t \rightarrow \infty$.

Theorems 2 and 4 provide a simple proof of the following familiar statement: If

$$
\int_{0}^{\infty}\|B(t)\| d t<\infty
$$

and all solutions of $\dot{x}=A x$ either (a) converge to 0 or are bounded or (b) diverge to $\infty$ as $t \rightarrow \infty$, then the same is true of (1.1). For (b) implies min $\Re\left\{\lambda_{i}(A)\right\}>0$. By a change of variable assume (1.1) is in the form $\dot{x}=\left\{T+S B(t) S^{-1}\right\} x$ with $T$ triangular,

$$
\int_{0}^{\infty}\left|\left\{S B(t) S^{-1}\right\}_{i j}\right| d t<\infty
$$

for all $(i, j)$ and $(2.16)$ holds,

$$
\liminf _{t \rightarrow \infty} \frac{1}{t} \int_{0}^{t} \min \Re\left(\left\{S B(s) S^{-1}\right\}_{i i}\right) d s=0,
$$

and (2.23) holds. Case (b) follows by Theorem 4. Case (a) is similar.

3. The equation (1.2). If $f(t)$ is a continuous complex-valued almostperiodic (a.p.) function on $[0, \infty)$ set

$$
M\{f(t)\}=\lim _{t \rightarrow \infty} \frac{1}{t} \int_{0}^{t} f(s) d s .
$$


A minor modification of an argument due to Favard (2) proves the following:

THEOREM 5. If $f(t)$ is real-valued, $M\{f(t)\} \geqslant 0$, and

$$
\int_{0}^{t} f(s) d s
$$

is not bounded on $[0, \infty)$, then there exists a sequence of intervals $\left[a_{n}, b_{n}\right]$ with $b_{n} \geqslant a_{n} \geqslant 0, a_{n}<a_{n+1}(n=0,1, \ldots), \lim a_{n}=\infty$, such that

$$
\int_{a_{n}}^{b_{n}} f(s) d s \geqslant n .
$$

We show in Theorem 6 that Theorem 5 is easily applied to obtain some sufficient conditions that imply the stability of (1.2) assuming the boundedness on $[0, \infty)$ of solutions to

$$
\dot{x}=A(t) x .
$$

For any finite collection of a.p. functions and any $\epsilon>0$ there exists a common relatively dense set of translation numbers with respect to $\epsilon$. Hence we may consider $A(t)$ an a.p. matrix function.

Denote by $X(t)$ the fundamental matrix of solutions (f.m.s.) of (3.1) with

$$
X(0)=I .
$$

Note that

$$
\limsup _{t \rightarrow \infty}\|X(t)\|<\infty
$$

and

$$
|X(t)|=\exp \left\{\int_{0}^{t} \operatorname{tr} A(s) d s\right\}
$$

together with the Hadamard determinant theorem imply that

$$
m(h)=\limsup _{t \rightarrow \infty} \int_{0}^{t} \Re \operatorname{tr} A(s+h) d s<\infty
$$

for any $h \geqslant 0$. We have

Theorem 6. Assume

$$
M\{\Re \operatorname{tr} A(t)\} \geqslant 0,
$$

$$
\text { (3.2) holds, }
$$

$$
\begin{aligned}
& \limsup _{h \rightarrow \infty} m(h)<\infty, \\
& \int_{0}^{\infty}\|B(s)\| d s<\infty ;
\end{aligned}
$$

then all solutions of (1.2) are uniformly bounded on $[0, \infty)$.

Before proceeding, note that (i) and (ii) imply $M\{\Re \operatorname{tr} A(t)\}=0$. 
Proof. First consider the translated equation

$$
\dot{x}=A(t+h) x
$$

(ii) clearly implies that all solutions of (3.5) are bounded on $[0, \infty)$ for each $h$.

Let $X(t ; h)$ be the f.m.s. of (3.5). Suppose there exists $h_{n} \rightarrow \infty$ such that

$$
\lim _{n \rightarrow \infty} \limsup _{t \rightarrow \infty} X\left(t ; h_{n}\right)=\infty \text {. }
$$

Then

$$
\lim _{n \rightarrow \infty} \limsup _{t \rightarrow \infty} \| X\left(t+h_{n}\right) \text { adj } X\left(h_{n}\right) \| \exp \left\{-\int_{0}^{h_{n}} \Re \operatorname{tr} A(s) d s\right\}=\infty,
$$

and we conclude from (ii) that

$$
\int_{0}^{t} \Re \operatorname{tr} A(s) d s
$$

is not bounded on $[0, \infty)$. By Theorem 5 there exists a sequence of intervals $\left[a_{n}, b_{n}\right]$ such that

$$
\int_{a_{n}}^{b_{n}} \Re \operatorname{tr} A(s) d s \geqslant n
$$

Setting $l_{n}=b_{n}-a_{n}$ and $s=a_{n}+t$, (3.7) becomes

$$
\int_{0}^{l_{n}} \Re \operatorname{tr} A\left(t+a_{n}\right) d t \geqslant n,
$$

and we conclude that $m\left(a_{n}\right) \geqslant n$, contradicting (iii). Hence there exists $K \geqslant 0$ such that

$$
\limsup _{h \rightarrow \infty} \limsup _{t \rightarrow \infty} X(t ; h)=K<\infty .
$$

Let $u(b, t)$ be a solution of $(1.2)$ with $u(b, 0)=b$. Using the variation of parameters formula and taking norms on both sides, we have

$$
\|u(b, t)\| \leqslant\|X(t)\|\|b\|+\int_{0}^{t}\left\|X(t) X^{-1}(s)\right\|\|B(s)\|\|u(b, s)\| d s .
$$

In (3.9) $t \geqslant s, t-s=h \geqslant 0$,

$$
\frac{d}{d s}\left\{X(s+h) X^{-1}(h)\right\}=A(s+h) X(s+h) X^{-1}(h)
$$

and it is obvious that $X(s+h) X^{-1}(h)$ is the f.m.s. of (3.5). By (3.8) we conclude that

$$
\limsup _{t \geqslant s \geqslant 0}\left\|X(t) X^{-1}(s)\right\|=K<\infty .
$$

Using an inequality due to Gronwall (3), we have

$$
\|u(b, t)\| \leqslant K\|b\| \exp \left(K \int_{0}^{t}\|B(s)\| d s\right)
$$

and (iv) completes the proof. 
We may remark that the argument applied to (3.5) will yield the usual stability theorem in case $A(t)$ is purely periodic without use of the Floquet representation of the f.m.s. as a product of exponential and periodic matrix functions.

\section{REFERENCES}

1. R. Bellman, Stability theory of differential equations (New York, 1953).

2. J. Favard, Sur les équations differentielles linéaires à coefficients presque-périodiques, Acta Mathematica, 51 (1927), 31-81.

3. T. H. Gronwall, Note on the derivatives with respect to a parameter of the solutions of a system of differential equations, Annals of Math., 20 (1918), 292-296.

4. V. I. Zubov, Some sufficient criteria for stability of a non-linear system of differential equations, Akad. Nauk. SSSR. Prikl. Mat. Meh. 17 (1953), 506-508; Math. Rev., 15 (1954), 126.

University of British Columbia 